\title{
Experimental Analysis and Investigation of WCDMA Link in Terms of Error Vector Magnitude Using Vector Signal Transceiver (VST) 5644R \& LabVIEW 2012
}

\author{
Gaurav Soni* \\ Associate Professor, Head-Center of Excellence ( $R \& D$ Wing) \\ Department of Electronics \& Communication Engineering \\ Amritsar College of Engineering \& Technology, Amritsar, Punjab, India \\ gaurav.ece@acetedu.in
}

\begin{abstract}
WCDMA is the most dominant $3^{\text {rd }}$ generation Cellular/Mobile Technology which is well known for providing wider bandwidth and high data rate for various mobile data and Voice services. In this work proposed WCDMA link is designed in LabVIEW 2012 and investigated on the hardware platform of VST 5644R experimentally. The modulation scheme used for the investigating WCDMA link on Vector Signal Transceiver 5644R (VST) platform is BPSK/QPSK. Here the results are measured and Evaluated in terms of EVM Value (Error Vector Magnitude). The objective of the proposed experimental based research is to design and optimize an WCDMA link using VST 5644R and evaluate the performance of the proposed WCDMA link at different system parameters like scrambling code, spreading factor, modulation scheme e.g.-QPSK, QAM.The results have shown that WCDMA Link based on QPSK scheme with 'long' scrambling code and scrambling factor of 128 performs much better as compare to that using 'short' scrambling code. By using VST device we check the real time performance of the WCDMA network on different value of the spreading factor like 64, 128. We are applying this spreading factor value on different modulation like BPSK, 16QAM with carrier frequency of $1800 \mathrm{MHz}$. However, simulation results show that the acceptable EVM value for WCDMA can be achieved with the use of high spreading factor with the complex modulation scheme. The experimental results of EVM obtained using BPSK/QPSK at spreading factor of 128 is $0.06 \%$ which claims maximum Signal to noise ratio (SNR) of $64.44 d B$ which shows the best and optimized WCDMA link performance which is claimed by our proposed experimental setup based on VST 5644R device.
\end{abstract}

Keywords: LabVIEW 2012, QPSK, Vector Signal Transceiver(VST), WCDMA

\section{Introduction}

Wideband Code Division Multiple Access (WCDMA) mobile technology has been evolved as the effective and widely accepted air interface technology for $3^{\text {rd }}$ generation Universal Mobile Telecommunication System (UMTS) network architecture[26-27]. Third generation system are designed for multimedia communication at high data rate. WCDMA technology is the most widely used third generation system which is spreading over a wide bandwidth by multiplying the user information with spreading sequence in WCDMA [2-5]. Due to increasing the demand of high data rate, more complex modulation schemes are used in various wireless communications resulting tightened error vector magnitude (EVM) requirement. For example, 64-QAM on LTE requires 8\%

${ }^{*}$ Corresponding Author 
of the minimum EVM level and 256-QAM on 802.11ac will adopt $2.5 \%$ of the EVM limit [10-12]. Error vector magnitude of system requires low value for complex modulation schemes. The error vector magnitude of system depends on the spreading factor. Spreading factor used to support high bit rate and mitigate the effect of inter-symbol interference. The main property of the spreading code is that they need to orthogonal to each other [3],[4-6]. The aim of this paper is to precisely evaluate the performance of WCDMA system with different spreading factor value.WCDMA is wideband digital cellular technology used for third Generation (3G) cellular communication. WCDMA is designed to allow many users to share the same RF channel by dynamically reallocating data rates and exactly match the demand of communication link of each user in the system[4],[15-26]. In WCDMA, each base transceiver station (BTS) output signal from all of its data channels multiplied with unique pseudo noise $(\mathrm{PN})$ code, referred to as a scrambling code. The users equipment (UE) receiver can differentiate one BTS from another by correlating scrambling code that unique provided to each BTS's. Similarly, each UE output signal is multiplied with a unique scrambling code that allows the BTS receiver to differentiate one UE from another. The scrambling code with fixed chip rate of $3.84 \mathrm{Mcps}$ is applied. The scrambling code is not independent code, the two user equipment having same scrambling code.The WCDMA radio link between BTS and UE have multiple data channels like video, packet data, bi-directional voice and background signalling messages, each represent a unique data channel with same carrier frequency. A BTS will transmit unique channels to many mobile users, and each mobile receiver distinguish it own channel from all other channels by using channelization codes, known as orthogonal variable spreading factor (OVSF) codes. Each channel originating from a BTS or from UE multiplied with different OVSF code [2], [6-7] also known as spreading factor. The length of OVSF code in WCDMA varies from 4 to 512 chips. The resulting downlink bitrate equal to system chip rate $(3.84 \mathrm{Mcps})$ divided by the spreading factor (SF). The scrambling codes allow reusing of spreading factor code among UE and BTS in same or different geographical area. The combination of scrambling code and spreading factor provide a unique communication channel between UE and BTS. During WCDMA communication, we used different modulation scheme like BPSK, QAM according to the transmission rate requirement. In BPSK modulation, they transmitting user data by using two phase of the carrier frequency. For example, if user data bit is ' 0 ' then they used $0^{0}$ phase of carrier signal. If user data bit is ' 1 ' they used $180^{\circ}$ out of phase of carrier signal. In QAM modulation, they generate output carrier frequency signal by varying phase with amplitude. In WCDMA we use 16QAM, 64QAM modulation. For high data rate communication, we used 64QAM, 16QAM modulation. For low data rate communication we used BPSK modulation. Two mode of communication used in WCDMA, Frequency division duplex (FDD) mode and Time division duplex (TDD) mode. For practically checking the performance of WCDMA, we use the VST (Vector Signal transceiver) 5644R device. The NI Vector Signal Transceiver combines radio frequency Input/output functionality in traditional box which contain Vector Signal Analyzers (VSAs) and Vector Signal Generator (VSGs) along with user defined functionality implemented on Field Programmable Gate Array (FPGA). The two independent local oscillators used for RF input and RF output coverage from $65 \mathrm{MHz}$ to $6 \mathrm{GHz}$ and bandwidth up to 200 $\mathrm{MHz}[5]$.The bandwidth requirement of WCDMA [8-10] is based on $5 \mathrm{MHz}$ wide RF radio signal and generally use chip rate of $3.84 \mathrm{Mcps}$. WCDMA technology is designed to be a high- performance system able to support high bandwidth and requiring simultaneous transmission of several bit streams that require different requirements of quality of service (QoS). In WCDMA based transmission it using two type of PN code- The first is called scrambling code and second type is called Orthogonal variable spreading factor (OVSF) code. Scrambling code provides unique identity to user equipments (UEs) and BTSs used in communication system. That BTS identify each user by its scrambling code and user equipment also identifies the BTSs by it scramble code. During transmission 
from the BTS to UE the data is decoded by applying scrambling code for that BTS were data actually originated and rejects another data that appear as noise at the receiver. When data come from UE to UE, at the receiver UE apply that scrambling code which was actually used at the transmitter UE and data is being decoded. In OVSF code can be used for identification of which type of communication occur between user equipment and BTS. Like for voice communication using OVSF1 code is used and for data communication OVSF 2 code [7] is used. The scrambling codes[4] are responsible for the most of the interference as these codes are not orthogonal. The channelization codes [6-9] may also result in interference as their orthogonality may be lost due to propagation environment. This work is based on experimental and real time implementation of WCDMA Link based on PXIe VST 5644 platform[1].The objective of the proposed experimental based research is to design and optimize an WCDMA link using VST 5644R and evaluate the performance of the proposed WCDMA link at different system parameters like scrambling code, spreading factor, modulation scheme e.g-QPSK, QAM. The rest of the paper is organized as follows- section 2 discusses the Vector Signal Transceiver- VST 5644R, section 3 demonstrates the experimentally set-up of the proposed WCDMA link, section 4 explains the results and followed by comprehensive conclusion.

\section{Brief Review of Vector Signal Transceiver (VST) 5644R}

To analyze and check the continuous feasibility of WCDMA signal, USA based company-National Instrument gives an effective device i.e., PXIe 5644R RF toolbox. National Instruments conveys quick, adaptable, and precise RF equipment and Lab View programming to meet the regularly changing requests of the remote business and see the building procedure through from configuration to approval to generation [1-14]. To keep pace with the continually RF development, LabVIEW graphical outline programming offers an arrangement of instruments for signal investigation, representation, and handling of standard and custom computerized and simple regulation configurations customized programming and adaptable equipment address an extensive variety of test needs including WLAN, GPS, WiMAX, MIMO, ZigBee, and RFID. This product planned methodology conveys the reliable and cost-decreasing remote test frameworks that architects have come to depend on from National Instruments .Engineers looking for another level of adaptability and execution in their test frameworks consolidate LabView with PXI RF particular instrumentation. These smaller, elite estimation frameworks highlight propelled timing and synchronization and the most recent PC advances, giving high throughput to estimations from DC to $6.6 \mathrm{GHz}$. With the NI estimation Suite for WCDMA. Consolidated with PXI RF instrumentation, these toolbox help PXI Express WCDMA estimation frameworks accomplish R\&D-grade precision and estimation times three to 10 times speedier than customary box instruments. Likewise, world-class RF instruments, for example, the NI PXIe-5644R RF vector signal analyzer [4] and NI PXIe5644R RF vector signal generator convey industry-driving estimation exactness at the cost. At last, you can utilize this product characterized test stage to test numerous remote benchmarks including GPS, WLAN, GSM/EDGE/WiMAX, and numerous others with the same RF equipment. One of the imperative components of this new VST is the capacity of clients to program the FPGA. Vector signal handset (VST) as of today is the greater part of the mechanized RF test frameworks user application programming to speak with these test instruments. Since RF applications are getting increasingly perplexing with time, specialists and researchers are investing huge amount to test an expanding measure of usefulness without expanding test times. To lessen test time, changes have been made in test calculations; transport speeds have expanded, and focal preparing unit speeds have been expanded; all things considered further enhancements are required to coordinate the expansion in the many-sided quality of RF test applications. [4] Real-time simulation 
instruments, for example-vector signal analyzer (VSA) and vector signal generator (VSG) are frequently used to estimate BER and EVM values effectively. In invulnerability tests, it is valuable to have a target set of measurements, for occasion BER as opposed to a subjective arrangement of measurements, for example, sitting tight for a LED to quit squinting or physically watching mutilations on a screen. A VSA consolidates an ongoing range analyzer and a constant oscilloscope. A VSG or a recurrence generator creates simple or computerized flags and is regularly utilized as a part of testing and investigating. For instance, while ascertaining BER one could just partition the aggregate number of blunder bits got by the aggregate number of bits got. VSG creates a digitally tweaked signal and the radio wire sends it into the resonation chamber. A short time later a VSA is utilized to demodulate the transmitted signal. To guarantee that the digitally adjusted sign is gotten without mistakes, the framework is aligned by associating a VSA and a VSG. This same trial setup permits us to decide the extent and period of the got flag and contrast it and the transmitted signal. This case likewise demonstrates that both the transmitter and beneficiary can be portrayed if an appropriately high information rate inspecting is utilized as a part of a signal era framework. NI as of late presented an answer, called a vector signal handset (VST), to address the requirement for testing rate and adaptability. A VST[4] is a little programming characterized RF framework that incorporates both a VSA and a VSG. This framework is bundled in an open industry standard, in particular a PXI module. The VST has a client programmable FPGA semiconductor chip installed in it to give programming designed circuits to constant signal preparing and control application purposes. This methodology permits a VST to have awesome adaptability, empowering it to supplant a few RF test instruments.

\section{Experimental Set Up \& Results}

Figure 1, shows the experimental arrangement for PXIe-1062 with VST 5644 used for generation, testing and analysis of WCDMA signals.

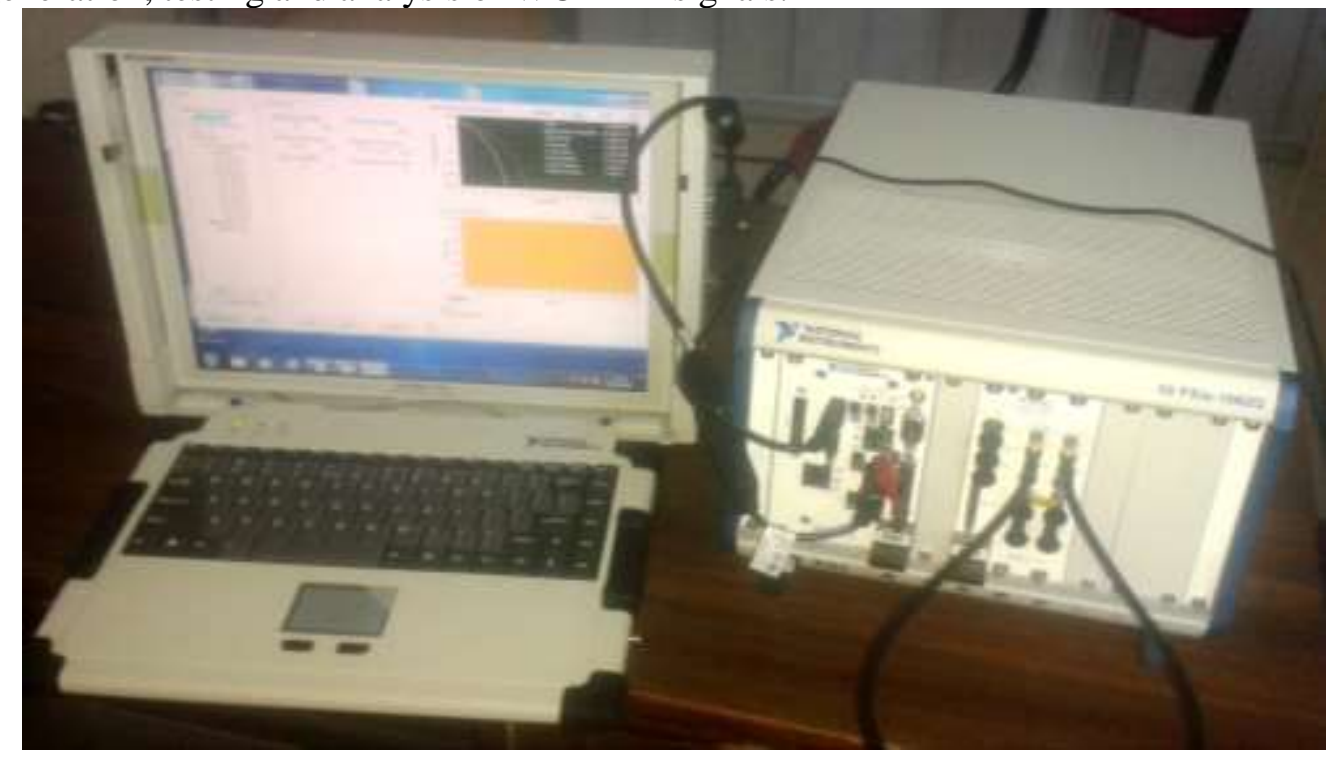

Figure 1. Experimental Set-up for WCDMA Link Based on NI Vector Signal Transceiver 5644R (Set-up \& Experiment Conducted at Center of Excellence- R \& D Wing, ACET, Amritsar)

WCDMA system incorporates some basic and important simulative parameters which may help achieve satisfactory performance of the WCDMA systems. These WCDMA parameters if not taken into account may also results in any wireless system to suffer degradation in terms of quality of service (QoS) link specifications like area coverage and 
capacity. In this experimental Work based on VST 5644R we tried to investigate some of these simulation parameters like spreading factor of WCDMA which affects performance of the system as a whole. Using VST 5644 at Physical Layer WCDMA Link [2-5] can be implemented. This implementation includes firstly decoding the downlink modulated WCDMA received signal, analyzing the received signal pattern in software LabVIEW 2012, calculating error vector magnitude (EVM) and relative input power. WCDMA is well known to provide variable user data rates i.e the Bandwidth on Demand (BoD) is being facilitated by WCDMA based system. The data rate capacity among the different users can vary from frame to frame. Here PXIe empowered by VST 5644R is used to generate and analyze the WCDMA based downlink signal. RF signal in WCDMA domain is generated by VST 5644. Scrambling codes are used as important parameter in both the link directions (uplink and downlink). Scrambling codes can be long range or short. Different unique modulation techniques QPSK with the different spreading factor for the different WCDMA channels used which further are required to improve the WCDMA system and the link range and capacity.

\section{Results \& Discussions}

Figure 2, shows the results of the proposed WCDMA Link based on LabVIEW 2012 designed using QPSK with 'short' scrambling code. The RMS EVM obtained for this scenario is $39.41 \%$ at spreading factor of 32 .

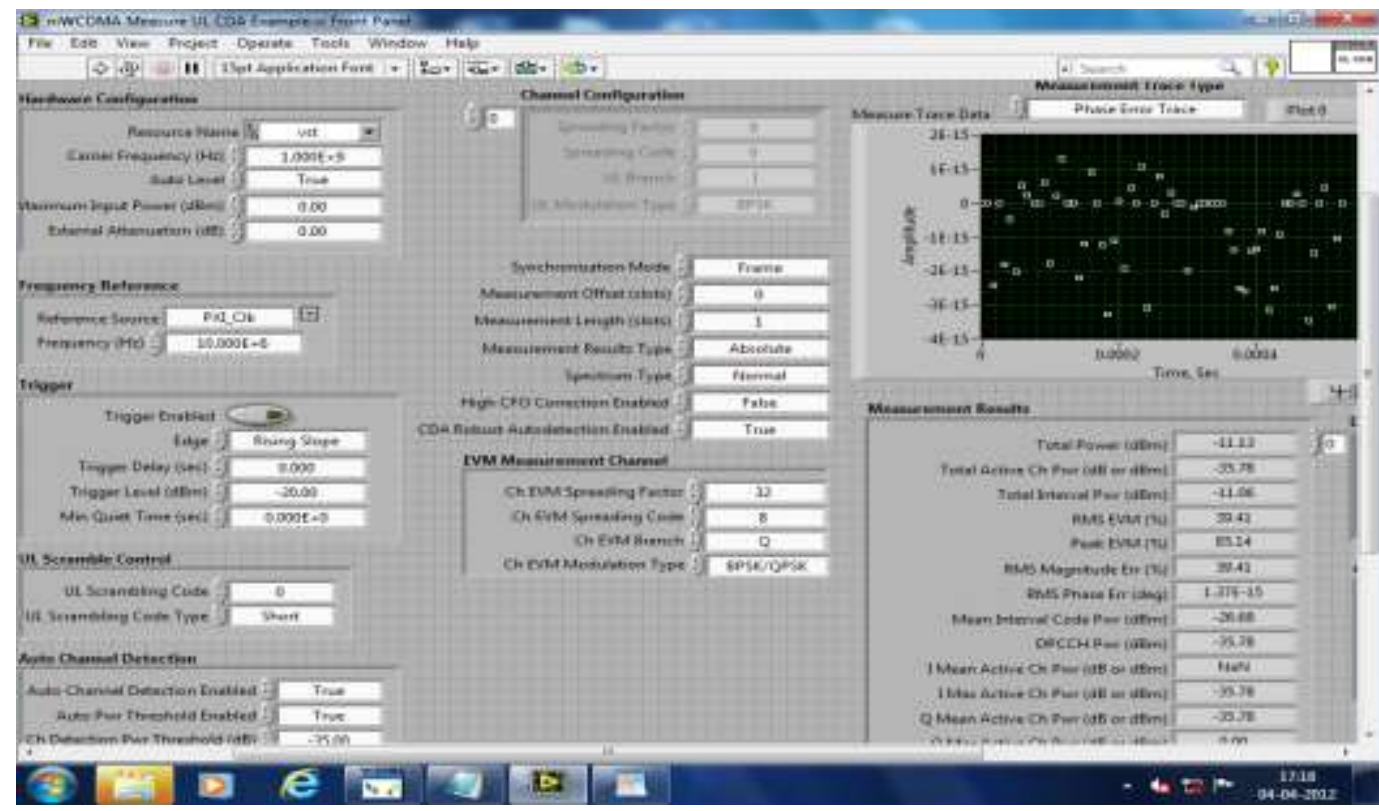

Figure 2. The WCDMA Based Simulation Parameters Along with the EVM using QPSK with (Short) Scrambling Code of 32

Figure 3, shows the simulation parameters and results in the form with the EVM diagram using QPSK at 'long' spreading code and spreading factor of 64.The performance analysis of WCDMA link is investigated based on EVM (Error Vector Magnitude). As shown in data of Figure 3, the EVM is $35.29 \%$. 


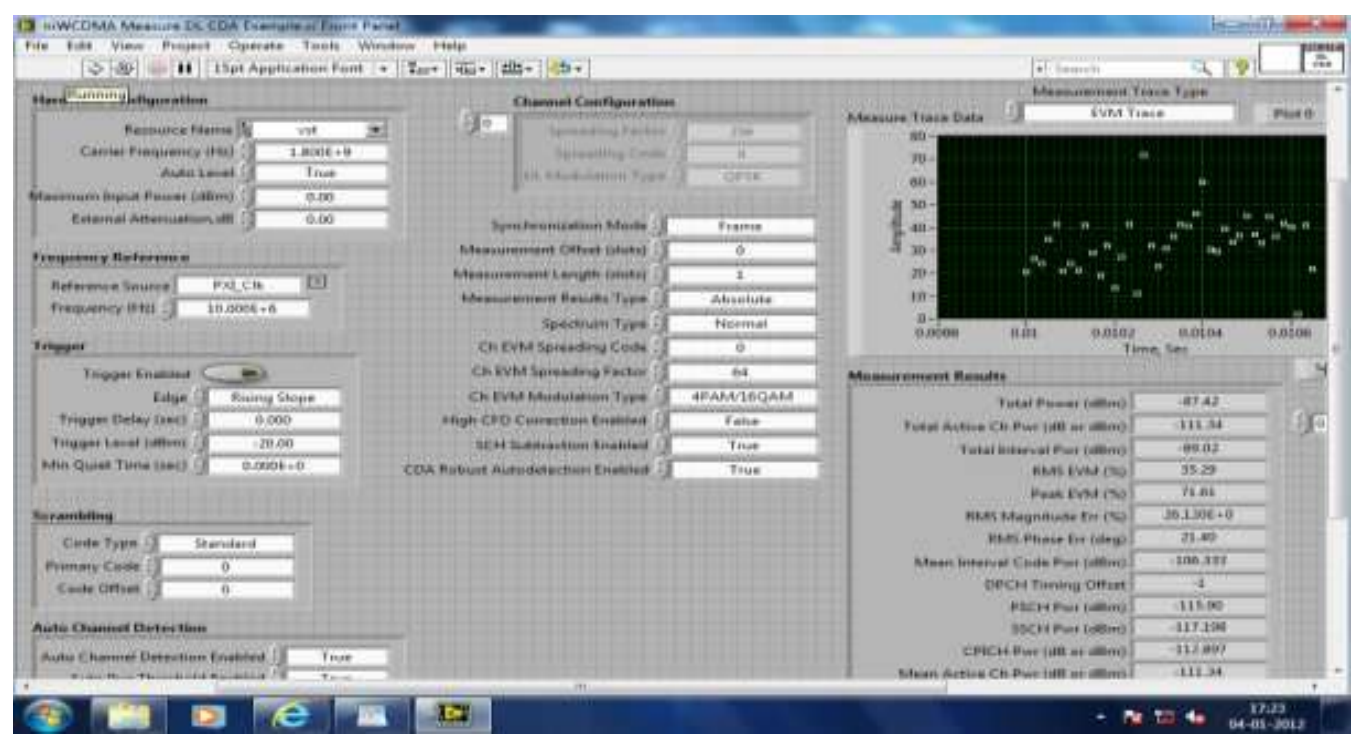

Figure 3. The WCDMA Simulation Parameters along with the EVM Value using 16 QAM with Long Scrambling Code and Spreading Factor of 64

Figure 4, shows the simulation parameters and results in the form with the EVM diagram using QPSK at 'long' spreading code and spreading factor of 128.The performance analysis of WCDMA link is investigated based on EVM (Error Vector Magnitude). As shown in data of figure 3 the EVM is $0.06 \%$ which clearly explains the acceptable link performance over the range WCDMA band of frequencies used.

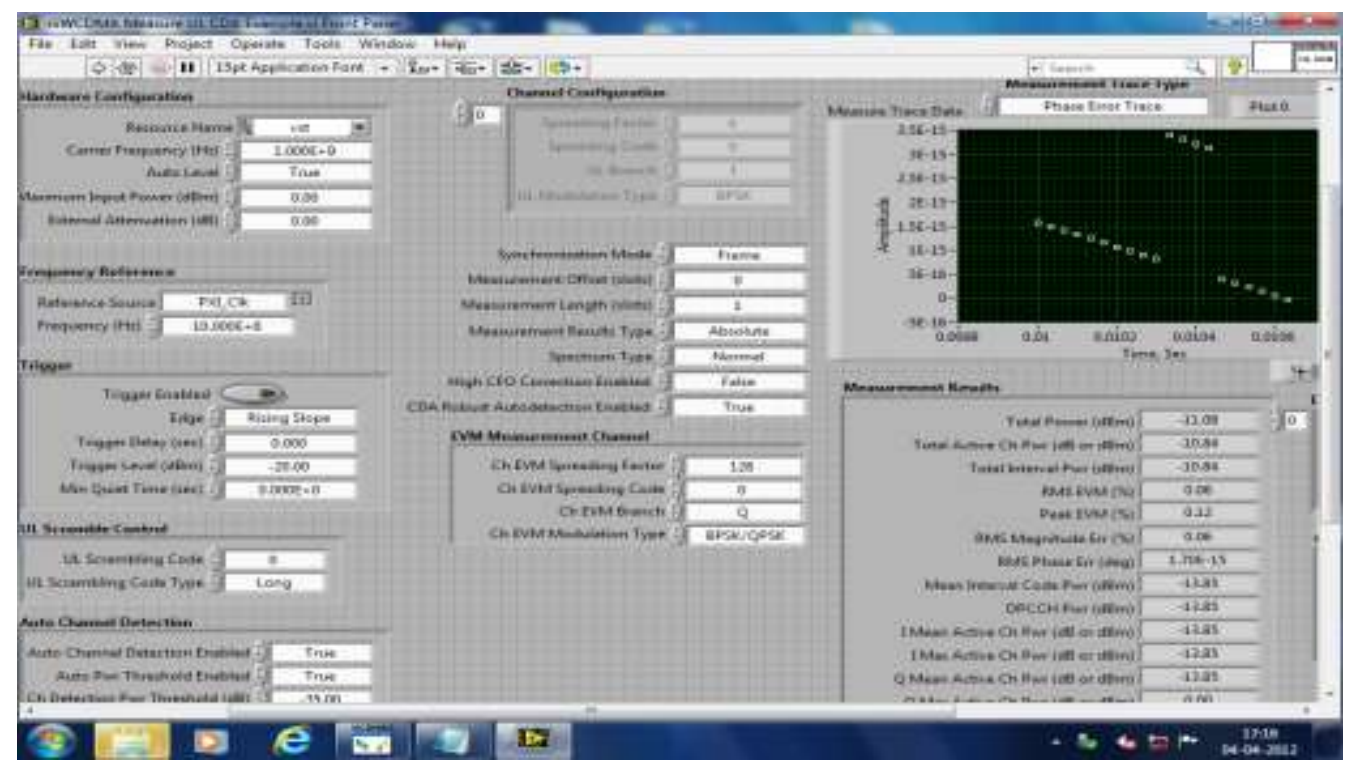

Figure 4. The WCDMA Simulation Parameters Along with the EVM Value using QPSK/BPSK with Long Scrambling Code and Scrambling Factor of 128

Figure 5, shows the simulation parameters and results in the form with the EVM diagram using 16 QAM and spreading factor of 128.The performance analysis of WCDMA link is investigated based on EVM (Error Vector Magnitude). As shown in data of figure 5 the EVM is $36.80 \%$. 


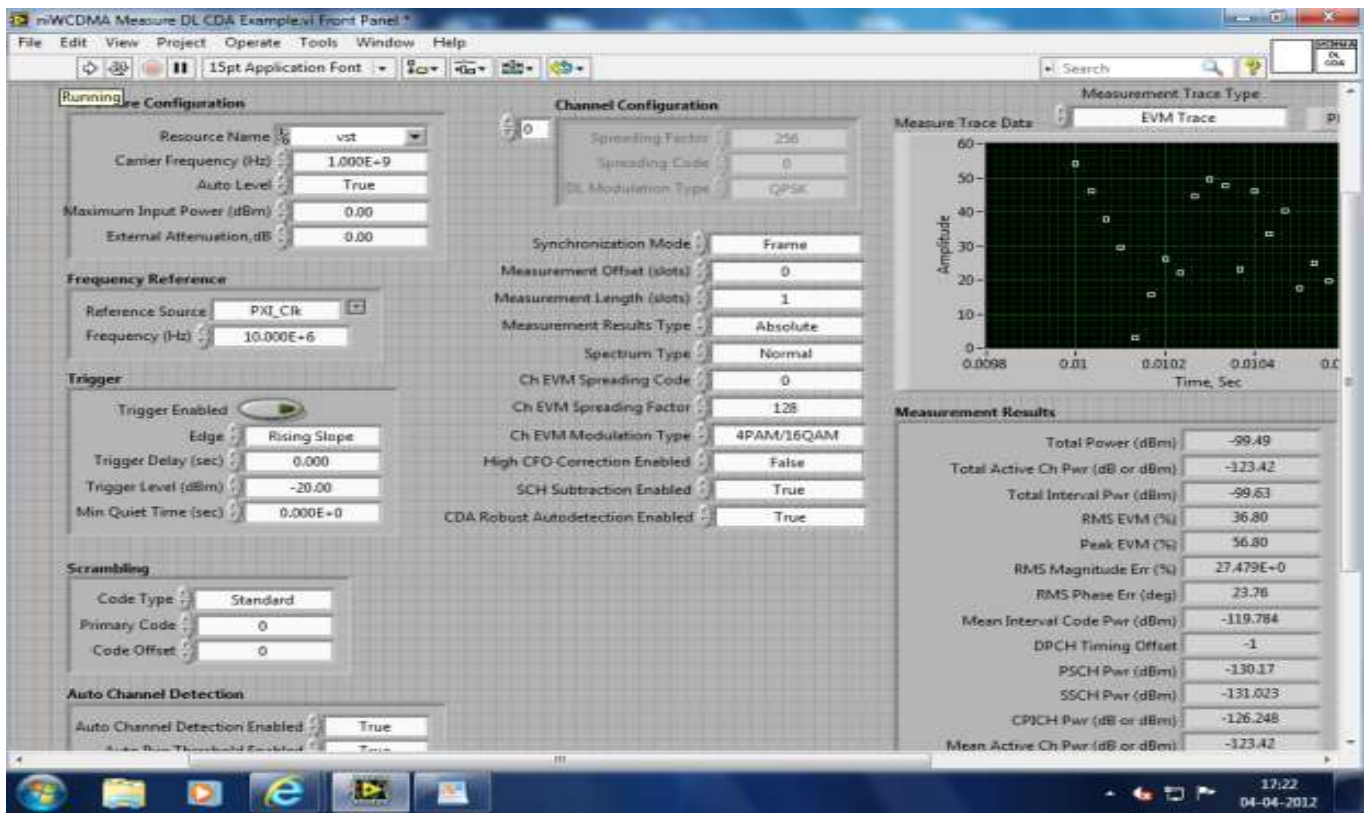

Figure 5. The WCDMA Simulation Parameters along with the EVM Value using 16QAM and Spreading Factor of 128

Table 1, shows the different values of EVM achieved at different values of spreading factor and modulation. The results in the table entries clearly shows that at spreading factor of 128 and under QPSK modulation scheme best performance is achieved which is being clear from the EVM value achieved which is .06 and corresponding SNR is 64.44 $\mathrm{dB}$. Further for the better understanding the link performance the EVM is converted to SNR using the following formula Equation (1):

Formula used for conversion of EVM to Signal to Noise Ratio (SNR):$S N R=39.3-20 \log _{10}[E V M \%] \mathrm{Db}$

\section{Table 1. Comparison of the Proposed Experimental Result with the Research Presented in the Previous Base Paper}

\begin{tabular}{|l|l|l|l|}
\hline $\begin{array}{l}\text { Modulation } \\
\text { Scheme }\end{array}$ & $\begin{array}{l}\text { Spreading } \\
\text { Factor }\end{array}$ & $\begin{array}{l}\text { Proposed } \\
\text { Research } \\
\text { Experimentally } \\
\text { EVM value } \\
\text { Achieved }(\%)\end{array}$ & $\begin{array}{l}\text { EVM value } \\
\text { converted to } \\
\text { SNR in dB }\end{array}$ \\
\hline QPSK & 32 & $39.41 \%$ & 8.09 \\
\hline 16 QAM & 64 & $35.29 \%$. & 9.05 \\
\hline QPSK & 128 & $0.06 \%$ & 64.44 \\
\hline 16 QAM & 128 & 36.80 & 8.68 \\
\hline
\end{tabular}

\section{Conclusion}

The LabVIEW 2012 based Wireless Communication WCDMA Application suite1.0 typically based on PXIe 1062Q empowered by VST 5644R WCDMA wireless measurement suite provides a real-time simulation environment and interface for WCDMA downlink running on National instruments based NI SDR hardware and software platform. In this article the NI PXIe-5644R vector signal transceiver Real time 
simulator is worked out for WCDMA to enable efficient, accurate, predictive, coded throughput results. The experimental work based on WCDMA have shown that EVM value increases significantly as the scrambling code is increased from short code stream to long code stream. QPSK with spreading factor 128 and 'long' spreading code yields best performance as EVM measured in this work is as low as $0.06 \%$. The experimental results of EVM obtained using BPSK/QPSK at spreading factor of 128 is $0.06 \%$ which claims achievement of maximum Signal to noise ratio (SNR) of $64.44 \mathrm{~dB}$ which shows the best ever WCDMA link performance claimed experimentally based on VST 5644R device.

\section{Acknowledgements}

The author would like to express his sincere thanks to the most respectful Mr. Amit Sharma (Honorable Chairman of Amritsar College of Engineering \& Technology, Amritsar, Punjab, UGC Autonomous college, NBA \& NAAC Accredited) for his whole hearted support and encouragement, sponsorship in developing the WCDMA based system and for carrying out this research work in the Center of Excellence - R and D wing which is being empowered by world class Sophisticated hardware like PXIe, VST 5644R and USRP 2920 and Licensed software LabVIEW 2012 from National Instruments, Bangalore. Further the author would extend gratitude for the co-operation and help for the successfully execution of this work in the Center of Excellence- Research and Development Wing ( $\mathrm{R}$ and $\mathrm{D}$ ) of Electronics and Communication Engineering Department, Amritsar College of Engineering \& Technology, Amritsar.

\section{References}

[1] G. Soni, "Performance Analysis of WCDMA Using Vector Signal Transceiver 5644R," 2016 Second International Conference on Computational Intelligence \& Communication Technology,(CICT), Ghaziabad,(2016), pp. 321-324.

[2] W. Yao and X. Yang, "Design of mobile terminal location system in WCDMA," 2016 16th International Symposium on Communications and Information Technologies (ISCIT), Qingdao, (2016), pp. 22-26.

[3] N. E. Mosilhy, M. S. El-Mahallawy and M. E. Khedr, "Multi-subcarrier OVSF code allocation in WCDMA," 2016 IEEE Canadian Conference on Electrical and Computer Engineering (CCECE), Vancouver, BC, (2016), pp.1-5.

[4] A. G. Markoc and G. Sisul, "Monitoring techniques at WCDMA radio network controller," 2016 International-Symposium-ELMAR,Zadar, (2016), pp. 5-8.

[5] M. Rahimi, G. Baghersalimi, M. R. Alizadeh, M. Najafi and N. B. Hassan, "BER assesment of a WCDMA-based Radio-over-Fiber system using a pilot-aided estimation and equalization approach," 2016 10th International Symposium on Communication Systems, Networks and Digital Signal Processing (CSNDSP), Prague, (2016), pp. 1-4.

[6] Zhenjie Deng, "Cross-network and cross-layer optimized video streaming over LTE and WCDMA downlink," 2016 IEEE Symposium on Computers and Communication (ISCC), Messina, (2016), pp. 868-873

[7] Balyan, D. S. Saini and R. Bansal, "Flexible fixed set portioning and performance improvement in OVSF based networks," 2016 International Conference on Computing, Communication and Automation (ICCCA), Greater-Noida,India, (2016), pp. 687-692.

[8] M. Korde, "Performance enhancement of slot synchronization in W-CDMA," 2016 International Conference on VLSI Systems, Architectures, Technology and Applications (VLSI-SATA), Bangalore, (2016), pp. 1-5.

[9] Y. Qiu, Y. Zhao and D. Li, "Centralized power control strategy for small packet service in UMTS," 2016 8th IEEE International Conference on Communication Software and Networks (ICCSN), Beijing, (2016), pp. 387-391.

[10] M. Hudlička, C. Lundström, D. A. Humphreys and I. Fatadin, "BER estimation from EVM for QPSK and 16-QAM coherent optical systems," 2016 IEEE 6th International Conference on Photonics (ICP), Kuching, (2016), pp. 1-3.

[11] M. Mohamad, R. Nilsson and J. Van de Beek, "Minimum-EVM N-continuous OFDM," 2016 IEEE International Conference on Communications (ICC), Kuala Lumpur, (2016), pp. 1-5.

[12] M. Ramakrishnan Kuppusamy, S. M. Bokhari and B. Marichala Anjaneyalu, "Error Vector Magnitude (EVM)-Based Constellation Combiner for Cooperative Relay Network," in IEEE Communications Letters, vol. 20, no. 2, (2016), pp. 304-307. 
[13] C. Ni and T. Jiang, "Minimizing the Error Vector Magnitude With Constrained Cubic Metric and Spectral Sidelobe in NC-OFDM-Based Cognitive Radio Systems," in IEEE Transactions on Vehicular Technology, vol. 66, no. 1, (2016), pp. 358-363.

[14] G. Soni, "Performance investigation of LTE systems based on NI PXIe-5644R vector signal transceiver", First IEEE international conference on Global Conference on, Communication Technologies (GCCT), 2 Thuckalay, (2015), pp. 755-760.

[15] F. Zhang, L. Xu, H. Zhang, X. Cheng and M. Mu, "A novel evaluation method of WCDMA RNC signaling carrying capacity," 2016 16th International Symposium on Communications and Information Technologies,(ISCIT), Qingdao, (2016), pp. 352-356.

[16] S. Zheng and H. C. Luong, "A WCDMA/WLAN Digital Polar Transmitter With Low-Noise ADPLL, Wideband PM/AM Modulator, and Linearized PA," in IEEE Journal of Solid-State Circuits, vol. 50, no. 7, (2016),pp.1645-1656.

[17] M. V. DeepakNair, R. Giofrè, L. Piazzon, P. Colantonio and F. M. Ghannouchi, "Effects of windowing based Crest Factor Reduction technique on digitally predistorted PAs for multicarrier WCDMA," 2015 IEEE International-Wireless-Symposium-(IWS2015), Shenzhen, (2015), pp.1-4.

[18] S. Maier, H. Schlesinger, G. Luz, D. Ferling, W. Kuebart and A. Pascht, "3GPP ACLR and EVM measurements for millimeter-wave wireless backhaul applications at 60GHz," 2015 IEEE Radio and Wireless Symposium(RWS),San-Diego,CA,(2015), pp.13-16.

[19] R. Bhadada and H. Mehta, "Performance investigation modeling for modulation employable in WCDMA over AWGN fading channel," 2nd International Conference on Emerging Technology Trends in Electronics, Communication and Networking (ET2ECN), Surat, (2014).

[20] K. H. Bilal, I. K. Eltahir and A. Babiker, "Performance evaluation of DS-WCDMA," International Conference on Computing, Electrical and Electronics Engineering (ICCEEE), 2013, Khartoum, (2013), pp. 316-318.

[21] X. Jia, J. Yang and X. Liu, "Study on the Characteristic of WCDMA Uplink Complex Spreading and Modulation," 2009 5th International Conference on Wireless Communications, Networking and Mobile Computing,Beijing, (2009), pp. 1-4.

[22] S. Parkvall, E. Englund, M. Lundevall and J. Torsner, "Evolving 3G mobile systems: broadband and broadcast services in WCDMA,"in IEEE-CommunicationsMagazine, vol. 44 ,no. 2, (2006), pp. 30-36.

[23] Dirk Staehle "On the Code and Soft Capacity of the UMTS FDD Downlink and the Capacity Increase by using a Secondary Scrambling" Report No. 413 (Master Thesis) April, (2007).

[24] D. Malladi, J. Damnjanovic, X. Zhang and S. Willenegger, "Introduction to WCDMA enhanced uplink," Fifth IEEE International Conference on 3G Mobile Communication Technologies, (2004), pp. 240-244.

[25] O. Prätor, C. Unger, A. Zoch and G. P. Fettweis "Performance of Adaptive Chip Equalization for the WCDMA Downlink in Fast Changing Environments" IEEE 7th Int. Symp. on Spread-Spectrum Tech. \& Appl., Prague, Czech Republic, (2002) Septemeber 2-5.

[26] H. Holma and A. Toskala, "WCDMA for UMTS, Radio Access For Third Generation Mobile Communication, John Wiley \& Sons, Ltd., Third Edition, (2004).

[27] M. Shafi, S. Ogose and T. Hattori, "WCDMA Radio Access Technology for Third Generation Mobile Communication," in Wireless Communications in the 21st Century, 1, Wiley-IEEE Press, (2002), pp. 351-377.

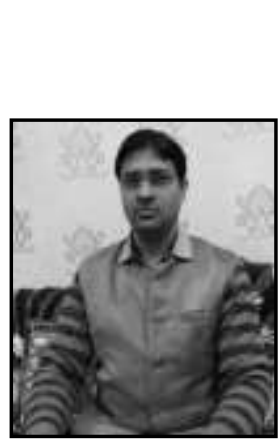

\section{Author}

Gaurav Soni, received his B-Tech Degree in Electronics and Communication Engineering from Punjab Technical University, Jalandhar in the year 2005 and achieved M-Tech Degree in Electronics and Communication Engineering with specialization in the field of optical and wireless communication from D.A.V.I.E.T, Jalandhar. He has more than ten years of teaching and research experience. He has to his credit more than 77 research papers in various Refereed International Journals indexed by Scopus-Elsevier and IEEE conference Proceedings and National Conferences. Presently, he is head of the Center of Excellence-Research and Development Laboratory of ECE, ACET, Amritsar. He has been exclusively involved in various Research oriented activities and in the capacity of the Prime Coordinator, he received Grants from AICTE, New Delhi, under MODROB Scheme in 2015-2016 worth INR 15 Lakhs and further made another Milestone by the Achievement of receiving FIST 2016 Grant approved By 
Department of Science and Technology, India (DST) worth INR 80 Lakhs for the establishment of Microwave Lab in the Department of Electronics and Communication Engineering, Amritsar College of Engineering and Technology, Amritsar (ACET). He is a member of the IEEE and ACM Society and life member of SPIE (Society of Photographic Instrumentation Engineers). He has served as Reviewer to IEEE-OSA Journal and a Prime Reviewer of various IEEE Conferences e.g- CSNT 2016, CICN 2016, etc. He has to his credits various Awards and honors received from various reputed research organizations from time to time. He made a great contribution to bring prestige and honor to his name and also to the institute Amritsar College of Engineering and Technology, Amritsar, to which he is presently serving. He investigated a highspeed optical transceivers and electrical-optical circuit board (EOCB) technology for chip-to-chip optical interconnects. He completed his master thesis in the latest field of research in Free Space Optical Communication. He designed a Free Space Optical Communication Link with speed of $2.5 \mathrm{Gbps}$. His research interests includes Wireless communications based system Design, next generation optical access networks, high-speed optical transmission systems, and Optical wireless communications system e.g., Free Space Optical communication systems. He is proud advocate of practical implementing a MIMO based Wireless RF Link using LabVIEW and USRP and VST (Vector Signal Transceiver 5644R) devices.

E-mail : gaurav.ece@acetedu.in , Mobile No. : +91-9781506102 